\title{
BER Estimation for HiperLAN/2
}

\author{
Lodewijk T. Smit, Gerard J.M. Smit, Johann L. Hurink, and \\ Gerard K. Rauwerda \\ Department of Electrical Engineering, Mathematics \& Computer Science \\ University of Twente, Enschede, the Netherlands \\ L.T.Smit@utwente.nl
}

\begin{abstract}
This paper presents a method to estimate the bit error rate (BER) of the wireless channel based on statistical analysis of the soft output of the receiver only. In HiperLAN/2 several modulation schemes can be used. The system should select the most suitable modulation scheme dependent on the quality of the wireless link and the Quality of Service requirements of the user. Our BER estimation method can be used to estimate the current quality of the wireless link and the quality when another modulation mode is considered. With this information, it is possible to select the most suitable modulation scheme for the current situation.
\end{abstract}

\section{Introduction}

This paper presents a method to estimate the bit error rate (BER) of the wireless channel based on statistical analysis of the soft output of the receiver. The method does not use pilot symbols and does not require knowledge of the properties of the channel.

The physical layer of HiperLAN/2 (and also IEEE 802.11a) can use four modulation schemes: BPSK, QPSK, 16QAM and 64QAM. A modulation scheme with more bits per symbol allows a higher throughput, but requires a better channel to receive the bits with the same quality.

HiperLAN/2 uses a forward error correction (FEC) Viterbi decoder after the receiver to correct the incorrectly received bits in a frame. Figure 1 depicts this configuration. In most cases, the Viterbi decoder can still correct a frame with a high BER (e.g. up to 10\%). It is attractive to switch to a modulation scheme with as many symbols per bit as possible because of the higher throughput, as long as the used Viterbi decoder is able to correct most of the received frames. To operate such a mechanism at run-time, an accurate estimation of the current wireless channel (BER) is required to select the optimal modulation mode. Furthermore, it would be nice to be able to predict what will occur with the current quality when we consider to change the modulation scheme. Our BER estimation method can be used to estimate the quality for the current modulation scheme and the quality when we consider to change to other modulation schemes. With this information, the control system in Figure 1 can select the best modulation scheme for the current situation and given the requested QoS. 
The paper is organized as follows. Section two describes related work. Section three describes a method to obtain detailed information about the quality of the wireless link. Instead of using pilot bits to obtain this quality information, we introduce another method based on statistical analysis of the received data. Section four evaluates the performance of this method. Section five discusses some implementation issues of our proposed method.

\section{Related Work}

Khun-jush [5] states that the Packet Error Rate (PER), determined using the checksum of a packet, is a suitable measure of the link performance. Although the PER gives an indication of the quality, this quality metric is rather coarse. Besides that, a slightly higher PER might be desirable compared to a lower PER with a considerable lower thoughput (e.g. due to another modulation scheme). A disadvantage is the introduced latency, because the PER should be calculated using enough packets to get an accurate estimation of the PER. Furthermore, it gives no indication what we could do to improve the current situation.

A commonly used method to estimate the quality of a wireless link is to compute the BER using pilot symbols. Pilot symbols represent a predefined sequence of symbols, which are known at the transmitter and the receiver side. Therefore, the BER can be computed from these pilot symbols. For example, HiperLAN/2 as well as third generation telephony uses pilot symbols [1]. This approach has two disadvantages. First, the transmission of the pilot symbols introduces overhead. Second, the BER is only computed over a small amount of the total bits that are transmitted.

Another approach is to model the channel with all the known effects, e.g. [7]. A state of the art article on this area is [3. Using this method it is possible to achieve accurate BER estimations for the modeled channel. However, the actual properties of the channel and the modeled effects can differ significantly from the constructed model. Also, effects that are not modeled can happen in real situations. In practice, it is not possible to model all the different effects that

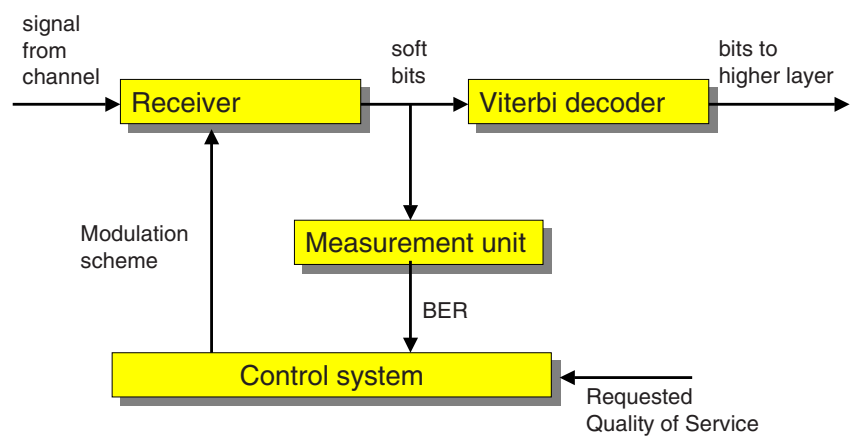

Fig. 1. The Control System of the HiperLAN/2 Terminal 
cause the disturbance of the wireless channel. Estimation of the exact quality of the signal of the wireless channel is therefore impossible.

Our approach differs significantly from the mentioned approaches. We only use the soft output from the receiver, and require no additional information about the channel. Furthermore, no pilot symbols are used. In our opinion, it does not matter which physical effect is responsible for the degradation of the signal to determine the BER. Therefore, information of the channel is not required to make an estimation of the BER. The advantage is that an accurate estimation can be made independent of the unpredictable dynamic changing external environment. Furthermore, our method provides the possibility to estimate the quality resulting from a planned adaptation of a parameter.

\section{BER Estimation}

This section explains how to estimate the BER of the output of the receiver so that we can predict the probability that the Viterbi decoder can correct a frame using the results of the previous section. Our method uses only the soft output of the receiver and thus no pilot symbols. Although pilot symbols are used for different purposes in HiperLAN/2 and therefore still need to be transmitted, this reason may be important for other applications of our BER estimation algorithm.

We start with an explanation of the method in detail for BPSK modulation followed by a shorter explanation of the method for the QPSK, 16-QAM and 64-QAM modulation schemes.

\subsection{BPSK}

In an ideal situation, without disturbance of the channel, the output of the soft value (also called symbol) of the receiver is equal to the transmitted symbol value. In case of BPSK modulation this means 1 or -1 . In case of disturbance of the channel, the sampled values are no longer exactly equal to 1 or -1 , but can be higher of lower. Figure 2 depicts this situation. A lot of external causes may be responsible for this disturbance. Most effects that change the signal can be modeled by a normal distribution. Other effects, e.g. fading, do not behave like a normal distribution. However, the central limit theorem 6] states that, if the number of samples is large $(>30)$, regardless of the type of the original distributions the resulting distribution is approximately a normal distribution. Therefore, we approximate the soft values of the output of the receiver with a normal distribution.

Figure 2 shows the expected normal distribution behavior for the soft output values of the receiver for a pretty good channel. When the channel becomes worse, the mean will not change (significantly), but the standard deviation will increase. Figure 3 shows the effect for an extremely bad channel. If all soft values $>0$ are considered to be transmitted ones and all soft values $<0$ are considered to be transmitted zeros, a lot of bits are received incorrectly in this figure. As can be seen from the figure, the two distributions are heavily mixed up. Every bit with value 1 that is received with a negative soft output is received badly 


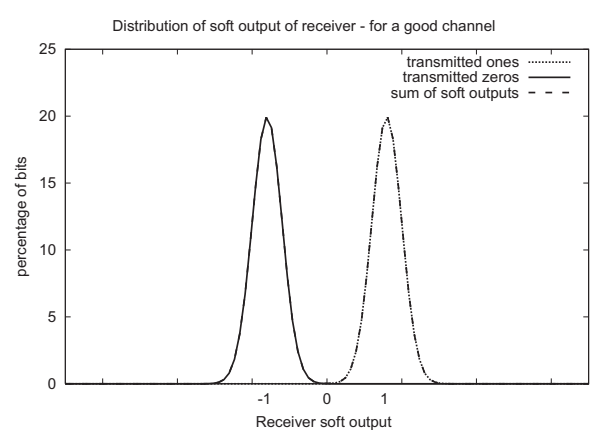

Fig. 2. Good Channel

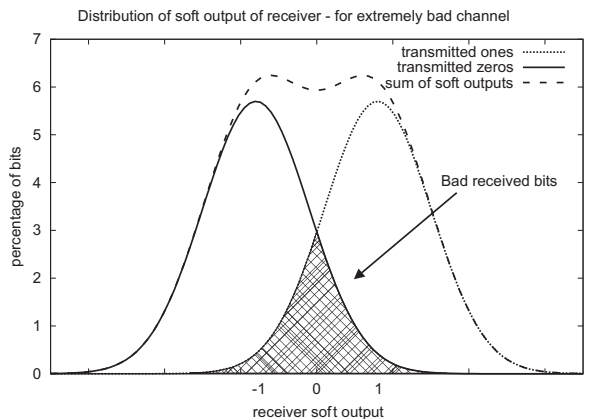

Fig. 3. Very Bad Channel

and also the positive soft output for a transmitted bit with value -1 is received badly. Thus, the marked area is the probability that a bit is received incorrectly.

Unfortunately, the receiver can not determine whether a soft value belongs to the 1-distribution or to the-1-distribution. The soft output of the receiver is the addition of the 1-distribution and the -1-distribution, which is also plotted in Figures 2 and 3 as a dotted line.

Our goal is to predict the bit error rate (BER), i.e. the size of the marked area in Figure 3. Let $\mathrm{X}(\mathrm{Y})$ denote the distribution of the soft output values of the transmitted -1 (1). Using these distributions, the BER can be expressed by:

$$
B E R=p P(X \geq 0)+(1-p) P(Y \leq 0) .
$$

where

$X$ : denotes the soft value of a transmitted -1 .

$Y$ : denotes the soft value of a transmitted 1 .

$p$ : denotes the probability that a -1 is transmitted.

Since both distributions are mirrored at the zero axis and due to the mentioned assumption that we can model these distributions with a normal distribution, $X$ and $Y$ can be expressed in terms of a standard normal distribution:

$$
\begin{aligned}
& X=\sigma Z-\mu . \\
& Y=\sigma Z+\mu .
\end{aligned}
$$

where $\mathrm{Z}$ denotes the standard normal distribution, $\mu$ the mean and $\sigma$ the standard deviation.

Using this, the BER reduces to:

$$
B E R=P(X \geq 0)=P\left(Z<\frac{\mu}{\sigma}\right)=\Phi\left(-\frac{\mu}{\sigma}\right) .
$$

where $\Phi(z)$ is the function that gives the area of the standard normal distribution to the right of $z$, i.e. the probability that a value is smaller than $z$. The function $\Phi(z)$ is widely available in tabular form. Note that $\Phi\left(-\frac{\mu}{\sigma}\right)$ is equal to $Q\left(\frac{\mu}{\sigma}\right)$, with $Q$ being the complementary error function that is commonly used in communication theory. 
To be able to calculate the BER via (4), we need good estimates for $\mu$ and $\sigma$. These estimates $\widehat{\mu}$ and $\widehat{\sigma}$ are derived using the soft output values of the receiver. As mentioned before, the received soft output values of the receiver do not correspond to the distribution $X$ and $Y$, but to a distribution $W$, which results from the combination of the distributions $X$ and $Y$ (with probability $p$ we get distribution $X$ and with probability $(1-p)$ distribution $Y)$. For $W$ we have:

$$
P(W \leq w)=p P(X \leq w)+(1-p) P(Y \leq w)
$$

Based on measured results for $W$ and using moments of distributions, it is possible to estimate the characteristic values $\mu$ and $\sigma$ of the distributions $X$ and $Y$, which together form distribution $W$ (see [10]). If $r$ is a positive integer, and if $X$ is a random variable, the $r$ th moment of $X$ is defined to be $m_{r}(X)=E\left(X^{r}\right)$, provided the expectation exists, see [4. For a standard normal distribution, the moments of $Z$ are shown in Table 1 The first and third moment of $Z$ are zero and can not be used to compute the two unknown variables $\widehat{\mu}$ and $\widehat{\sigma}$. Therefore the second and fourth moment of $W$ are used.

Table 1. Moments of Z

$$
\begin{array}{|l|l|}
\hline m_{1}(Z) & 0 \\
m_{2}(Z) & 1 \\
m_{3}(Z) & 0 \\
m_{4}(Z) & 3 \\
\hline
\end{array}
$$

The second moment of $W$ is:

$$
m_{2}(W)=p\left(E\left(X^{2}\right)\right)+(1-p)\left(E\left(Y^{2}\right)\right) .
$$

The scrambling used in HiperLAN/2 ensures that approximately an equal number of ones and zeros are transmitted. This means that $p \approx \frac{1}{2}$. Setting $p=\frac{1}{2}$, and using equations (2), (3) and Table 1, equation (6) becomes:

$$
m_{2}(W)=\mu^{2}+\sigma^{2} .
$$

therefore,

$$
\widehat{\sigma}^{2}=m_{2}(W)-\widehat{\mu}^{2} .
$$

The fourth moment of $W$ is:

$$
m_{4}(W)=p\left(E\left(X^{4}\right)\right)+(1-p)\left(E\left(Y^{4}\right)\right) .
$$

With $\mathrm{p}=\frac{1}{2}$, this equation becomes:

$$
m_{4}(W)=\mu^{4}+\left(\begin{array}{l}
4 \\
2
\end{array}\right) \mu^{2} \sigma^{2} E\left(Z^{2}\right)+\sigma^{4} E\left(Z^{4}\right) .
$$

Substituting the moments of $\mathrm{Z}$ gives:

$$
m_{4}(W)=\mu^{4}+6 \mu^{2} \sigma^{2}+3 \sigma^{4} .
$$


Replacing $\sigma^{2}$ with (7) and simplifying yields:

$$
\mu^{4}=\frac{3}{2}\left(m_{2}(W)\right)^{2}-\frac{1}{2} m_{4}(W)
$$

So,

$$
\mu=\sqrt[4]{\frac{3}{2}\left(m_{2}(W)\right)^{2}-\frac{1}{2} m_{4}(W)} .
$$

With the moment estimators of equations (8) and (14), the mean $\widehat{\mu}$ and standard deviation $\widehat{\sigma}$ can be computed from the individual samples $W_{1} . . W_{n}$ by:

$$
\begin{aligned}
& \widehat{\mu}=\sqrt[4]{\frac{3\left(\frac{\sum_{i=1}^{n} W_{i}^{2}}{n}\right)^{2}-\frac{\sum_{i=1}^{n} W_{i}^{4}}{n}}{c_{1}}} \\
& \widehat{\sigma}=\sqrt{\frac{\sum_{i=1}^{n} W_{i}^{2}}{n}-c_{2} \widehat{\mu}^{2}}
\end{aligned}
$$

where $c_{1}=2, c_{2}=1$ and $c_{3}=12$. We have introduced these constants to be able to use the same formulas for the other modulation schemes.

Sometimes, there exists no (real) solution for $\mu$ and/or $\sigma$. In this case $\mu^{4}$ and/or $\sigma^{2}$ in Equations (12) and (8) respectively are negative. So, this should always be checked before $\mu$ and $\sigma$ are computed.

Finally, the BER estimation can be computed with:

$$
\widehat{B E R}=\frac{c_{3}}{12} \Phi\left(-\frac{\widehat{\mu}}{\widehat{\sigma}}\right)
$$

\subsection{Extention to QPSK, 16-QAM, and 64-QAM Modulation Schemes}

HiperLAN/2 allows four modulation schemes: BPSK, QPSK, 16QAM and 64QAM. Therefore, the method for BER estimation for BPSK and QPSK schemes, that was presented in Chapter 3.1, should be extended for 16QAM and 64QAM modulation schemes. The 16QAM modulation scheme uses complex symbols. The real as well as the imaginary part of the complex symbol can have four different values: $-3,-1,1$ and 3 . Therefore, the complex symbol can have 16 values, representing 4 bits. Figure 4 shows the possible complex values of a transmitted symbol for 16QAM.

The 64QAM modulation scheme uses also complex symbols. The real as well as the imaginary part of the complex symbol can have eight different values: $-7,-5,-3,-1,1,3,5$ and 7 . Therefore, the complex symbol can have 64 values, representing 6 bits. 
Section 3.3 describes the derivation of the estimators for $\mu$ and $\sigma$ for 16QAM modulation. These estimators will be used in Section 3.4 to derive an estimator for the BER for 16QAM modulation. Similarly, section 3.5 describes the derivation of the estimators for $\mu$ and $\sigma$ for 64QAM modulation and Section 3.6 derives an estimator for the BER for 64QAM modulation.

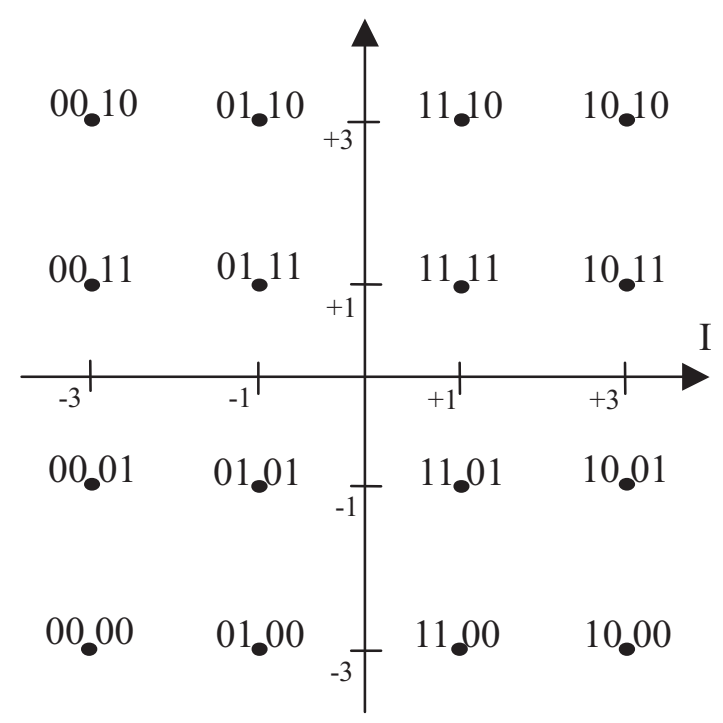

Fig. 4. 16-QAM Constellation Bit Encoding Scheme

\subsection{Estimators for 16QAM}

Figure 4 shows the possible complex values of a transmitted symbol for 16QAM. We consider the real and the complex part separate, because they are independent. So, the real part of a symbol that is transmitted can have four different values $(-3,-1,1$ and 3$)$, compared to two possible values $(-1,1)$ in the UMTS case with BPSK/QPSK modulation. Therefore, new estimators should be derived for $\widehat{\mu}$ and $\widehat{\sigma}$.

First, we define four stochastic variables for the four possible values, with:

$\mathrm{X}$ : denotes the distribution of the soft values of the transmitted minus ones.

$\mathrm{Y}$ : denotes the distribution of the soft values of the transmitted ones.

Q: denotes the distribution of the soft values of the transmitted minus threes.

$\mathrm{R}$ : denotes the distribution of the soft values of the transmitted threes.

We assume that the distribution have the same variance $\sigma$ and that the means are $-\mu, \mu,-3 \mu$ and $3 \mu$ respectively. Using this, the stochastic variables $\mathrm{X}, \mathrm{Y}, \mathrm{Q}$ and $\mathrm{R}$ are expressed in terms of a standard normal distribution $Z$ :

$$
X=\sigma Z-\mu \text {. }
$$




$$
\begin{aligned}
& Y=\sigma Z+\mu . \\
& Q=\sigma Z-3 \mu . \\
& R=\sigma Z+3 \mu .
\end{aligned}
$$

The received output values of the receiver do not correspond to these four individual distributions, but to a distribution $V$, which results from the combination of the four distributions. We assume that these four separate stochastic variables occur with the same frequency. In other words, the probability for each value is equal to $\frac{1}{4}$. For HiperLAN/2 the scrambling ensures that this property is fulfilled. For $V$ we have:

$$
P(V<v)=\frac{1}{4}(P(X<v)+P(Y<v)+P(Q<v)+P(R<v)) .
$$

Based on measured results of $V$ and using the moments of the distributions, it is possible to estimate the characteristic values $\mu$ and $\sigma$ of the four distributions, which form together distribution $V$.

The second moment $m_{2}$ of $V$ is:

$$
m_{2}(V)=\frac{1}{4}\left(E\left(X^{2}\right)+E\left(Y^{2}\right)+E\left(Q^{2}\right)+E\left(R^{2}\right)\right) .
$$

Using the following expressions for the second moments of the stochastic variable $\mathrm{X}, \mathrm{Y}, \mathrm{Q}$ and $\mathrm{R}$

$$
\begin{aligned}
& m_{2}(X)=E\left(X^{2}\right)=\sigma^{2}+\mu^{2} . \\
& m_{2}(Y)=E\left(Y^{2}\right)=\sigma^{2}+\mu^{2} . \\
& m_{2}(Q)=E\left(Q^{2}\right)=\sigma^{2}+9 \mu^{2} . \\
& m_{2}(R)=E\left(R^{2}\right)=\sigma^{2}+9 \mu^{2},
\end{aligned}
$$

the second moment of $V$ becomes:

$$
m_{2}(V)=\sigma^{2}+5 \mu^{2} .
$$

This results in the following estimator for the variance:

$$
\widehat{\sigma}^{2}=m_{2}(V)-5 \mu^{2} .
$$

To get an estimator for the mean $\mu$, we again use the fourth moment of $\mathrm{V}$ :

$$
m_{4}(V)=\frac{1}{4}\left(E\left(X^{4}\right)+E\left(Y^{4}\right)+E\left(Q^{4}\right)+E\left(R^{4}\right)\right) .
$$

Using the fourth moments of the different stochastic variables (the computation of the fourth moments is done in a similar way as in Section 3.1

$$
\begin{aligned}
& m_{4}(X)=E\left(X^{4}\right)=E\left((\sigma Z-\mu)^{4}\right)=\mu^{4}+6 \sigma^{2} \mu^{2}+3 \sigma^{4} \\
& m_{4}(Y)=E\left(Y^{4}\right)=E\left((\sigma Z+\mu)^{4}\right)=\mu^{4}+6 \sigma^{2} \mu^{2}+3 \sigma^{4} \\
& m_{4}(Q)=E\left(Q^{4}\right)=E\left((\sigma Z-3 \mu)^{4}\right)=81 \mu^{4}+54 \sigma^{2} \mu^{2}+3 \sigma^{4} \\
& m_{4}(R)=E\left(R^{4}\right)=E\left((\sigma Z+3 \mu)^{4}\right)=81 \mu^{4}+54 \sigma^{2} \mu^{2}+3 \sigma^{4},
\end{aligned}
$$


the fourth moment $m_{4}$ of $V$ becomes:

$$
m_{4}(V)=41 \mu^{4}+30 \sigma^{2} \mu^{2}+3 \sigma^{4} .
$$

With substitution of $\widehat{\sigma}$ in $m_{4}(V)$, we derive an estimator for $\mu$ :

$$
\widehat{\mu}=\sqrt[4]{\frac{3 m_{2}(V)^{2}-m_{4}(V)}{34}}
$$

With substitution of $\widehat{\mu}$ in $m_{2}(V)$, the estimator for $\sigma$ becomes:

$$
\widehat{\sigma}=\sqrt{m_{2}(V)-\widehat{\mu}^{2}} .
$$

With this derivations, estimators $\widehat{\sigma}$ and $\widehat{\mu}$ can be computed from the individual samples $V_{1} \ldots V_{n}$ by:

$$
\begin{aligned}
& \widehat{\mu}=\sqrt[4]{\frac{3\left(\frac{\sum_{i=1}^{n} V_{i}^{2}}{n}\right)^{2}-\frac{\sum_{i=1}^{n} V_{i}^{4}}{n}}{34}} \\
& \widehat{\sigma}=\sqrt{\frac{\sum_{\frac{i=1}{n} V_{i}^{2}}^{n}-5 \widehat{\mu}^{2}}{n}}
\end{aligned}
$$

Simulations within Matlab show that both estimators work well if the four individual distributions are normal distributions.

\subsection{BER Estimation for 16QAM}

A received symbol is mapped to the nearest symbol in the bit constellation encoding diagram for 16QAM (see Figure 4). This symbol may be another symbol than the transmitted symbol resulting in one or more bit errors.

Based on the estimators $\widehat{\mu}$ and $\widehat{\sigma}$ we derive an estimation of the bit error rate $\widehat{B E R}$. First, we investigate the error probabilities in one dimension. Figure 5 shows the four distributions of the four stochastic variables. The probability that the real part of a complex transmitted symbol with value $(\mathrm{i}, \mathrm{q})$ is received wrong is denoted by: $P_{e_{-} r e(i)}$.

These probabilities are given by:

$$
\begin{aligned}
P_{e \_r e(-3)} & =P(Q \geq-2) \\
P_{e \_r e(-1)} & =P(X \leq-2)+P(X \geq 0) \\
P_{e \_r e(1)} & =P(Y \leq 0)+P(Y \geq 2) \\
P_{e \_r e(3)} & =P(R \leq 2),
\end{aligned}
$$


and can be calculated by using the mean $\mu$ and the standard deviation $\sigma$ :

$$
\begin{aligned}
P_{e_{-} r e(-3)} & =\Phi\left(\frac{-\mu}{\sigma}\right) \\
P_{e_{-} r e(-1)} & =2 * \Phi\left(\frac{-\mu}{\sigma}\right) \\
P_{e_{-} r e(1)} & =2 * \Phi\left(\frac{-\mu}{\sigma}\right) \\
P_{e_{-} r e(3)} & =\Phi\left(\frac{-\mu}{\sigma}\right)
\end{aligned}
$$

The probability $P_{e_{-} r e}$ that the real part of a symbol is received incorrect is now given by:

$$
P_{e_{-} r e}=\frac{1}{4} P_{e_{-} r e(-3)}+\frac{1}{4} P_{e_{-} r e(-1)}+\frac{1}{4} P_{e_{-} r e(1)}+\frac{1}{4} P_{e_{-} r e(3)}=\frac{6}{4} \Phi\left(-\frac{\mu}{\sigma}\right) .
$$

The same holds for the probability $P_{e_{-} i m}$ for the imaginary part of the symbol.
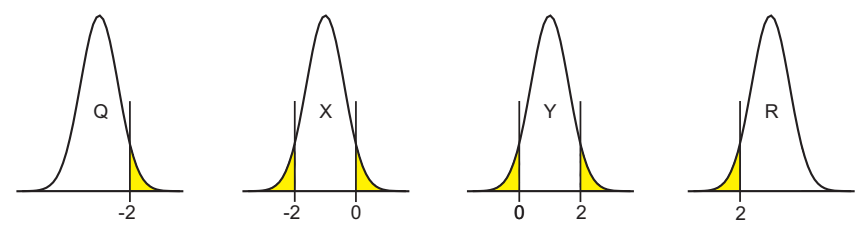

Fig. 5. BER Areas for 16-QAM Modulation Scheme in One Dimension

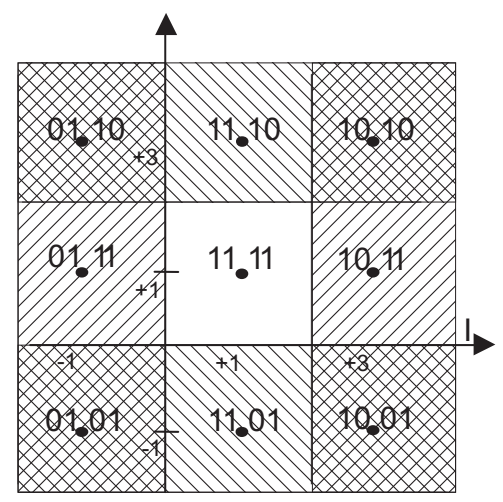

Fig. 6. Bit Error Probability for '1111' for 16-QAM

To calculate the BER, the mapping between symbol and bit sequence has to be considered. Figure 4 shows this mapping. Due to the used Gray coding, 
only one out of four bits is in error, if one of the direct neighbor symbols (of the transmitted symbol) is received in one dimension. If a symbol is received incorrectly, we assume that instead of the correct symbol, one of the direct neighbors is received. So, we assume that an incorrectly received real or complex part of a symbol leads to precisely one bit error. This assumption however, introduces a small estimation error. For now this error is ignored. In 8 this small error is investigated. affects the estimation. The probability of a complex bit error (in two dimensions) is the addition of the real part and the complex part. Note that the overlap of the two probability areas are counted twice. This is correct, due to the fact that indeed two bits are incorrect, instead of one. Figure 6] shows this effect for the reception of symbol '1111'. The probability of a bit error becomes:

$$
\begin{aligned}
\widehat{B E R} & =\left(P_{e_{-} r e}+P_{e_{i} i m}\right) /(\text { number of bits per symbol }) \\
& =\left(\frac{6}{4} \Phi\left(-\frac{\widehat{\mu}}{\widehat{\sigma}}\right)+\frac{6}{4} \Phi\left(-\frac{\widehat{\mu}}{\widehat{\sigma}}\right)\right) / 4 \\
& =\frac{3}{4} \Phi\left(-\frac{\widehat{\mu}}{\widehat{\sigma}}\right)
\end{aligned}
$$

\subsection{Estimators for 64QAM}

The 64QAM modulation scheme uses also complex symbols. The real as well as the imaginary part of the complex symbol can have eight different values: - $7,-$ $5,-3,-1,1,3,5$ and 7 . We consider the real and the complex part separate, because they are independent. So, the real part of a symbol that is transmitted can have eight different values $(-7 \mu,-5 \mu,-3 \mu,-1 \mu, 1 \mu, 3 \mu, 5 \mu$ and $7 \mu)$, compared to four values for the 16QAM case.

The derivation of the estimators for 64QAM can be done in the same way as for BPSK (QPSK) and 16QAM. Therefore, we leave out all intermediate steps. We define $W$ as the distribution that results from the combination of the eight separate normal distribution in one dimension with the eight means:-7,-5,-3,$1,1,3,5,7$. The second and fourth moment of $W$ are:

$$
\begin{aligned}
& m_{2}(W)=\sigma^{2}+21 \mu^{2} \\
& m_{4}(W)=777 \mu^{4}+126 \sigma^{2} \mu^{2}+3 \sigma^{4}
\end{aligned}
$$

With these moments, the estimators $\widehat{\sigma}$ and $\widehat{\mu}$ are as follows:

$$
\widehat{\mu}=\sqrt[4]{\frac{3\left(\frac{\sum_{i=1}^{n} W_{i}^{2}}{n}\right)^{2}-\frac{\sum_{i=1}^{n} W_{i}^{4}}{n}}{546}}
$$




$$
\widehat{\sigma}=\sqrt{\frac{\sum_{i=1}^{n} W_{i}^{2}}{n}-21 \widehat{\mu}^{2}}
$$

where $W_{1} \ldots W_{n}$ denote the $n$ individual samples.

\subsection{BER Estimation for 64QAM}

In a similar was as done in Section 3.4, we can derive the probability of a bit error for 64-QAM modulation:

$$
\begin{aligned}
\widehat{B E R} & =\left(P_{e_{-} r e}+P_{e_{-} i m}\right) /(\text { number of bits per symbol }) \\
& =\left(\frac{14}{8} \Phi\left(-\frac{\widehat{\mu}}{\widehat{\sigma}}\right)+\frac{14}{8} \Phi\left(-\frac{\widehat{\mu}}{\widehat{\sigma}}\right)\right) / 6 \\
& =\frac{7}{12} \Phi\left(-\frac{\widehat{\mu}}{\widehat{\sigma}}\right)
\end{aligned}
$$

\subsection{Summary for BPSK, QPSK, 16-QAM, and 64-QAM Modulation Scheme}

The BER estimation for the different types of modulation differs only by the constants $c_{1}, c_{2}$ and $c_{3}$ in the Formulas (14) to (16). Table 2 summarizes the constants for the different types of modulation that should be used for the Formulas (14) to (16). It may be a bit surprisingly that also in the BER estimation with Formula (16) the function $\Phi$ has to be weighted. This results from the fact that symbols that have neighbour symbols on both sides have a higher probability on a bit error.

Table 2. Constants for Different Modulation Schemes

\begin{tabular}{l|r|r|r} 
& $c_{1}$ & $c_{2}$ & $c_{3}$ \\
\hline BPSK & 2 & 1 & 12 \\
QPSK & 2 & 1 & 12 \\
16-QAM & 34 & 5 & 9 \\
64-QAM & 546 & 21 & 7
\end{tabular}

\section{Results BER Estimation}

We have chosen to show the test results of the BER estimation method for the 64-QAM modulation scheme. More bits per symbol makes the BER estimation more difficult. For these tests we used a HiperLAN/2 simulator [11. Figure 8 shows the results for the BER estimation for 64-QAM modulation, which is the most difficult case. The figure shows the BER on the $\mathrm{x}$-axis and the error in the 
estimation on the y-axis. The estimation error is equal to the (estimated BER - real BER) * $100 \%$. The line with label " 1 term" shows the results obtained by using the Formulas (14) to (16) and Table 2 As can be seen from the figure, there is an under estimation when the BER increases. The reason is that Formula (16) accounts for 1 bit error only when the received symbol is not the transmitted symbol. However, 16-QAM and 64-QAM modulation schemes are not modeled with two normal distributions, but with four or eight distributions respectively. This means that a symbol error also can be caused by two interfering distributions that are not directly next to each other, resulting in more bit errors. For example, if the received symbol is the neighbor of the neighbor of the transmitted symbol (denoted with distance 2), then it will account for 2 bit errors, as can be seen from Table 3 We can correct for this case with the addition of an additional term to Formula (16). This term is the probability that a symbol has neighbors with distance 2 multiplied with the error introduced if indeed a neighbor with distance 2 is received instead of the correct symbol. So, the correction term for a received neighbor with distance 2 is: $\frac{6}{12}\left(\Phi\left(\frac{5 \mu}{\sigma}\right)-\Phi\left(\frac{3 \mu}{\sigma}\right)\right)$, see Figure 7 A similarly correction term for a received neighbor with distance 3 is: $\frac{5}{12}\left(\Phi\left(\frac{7 \mu}{\sigma}\right)-\Phi\left(\frac{5 \mu}{\sigma}\right)\right)$.

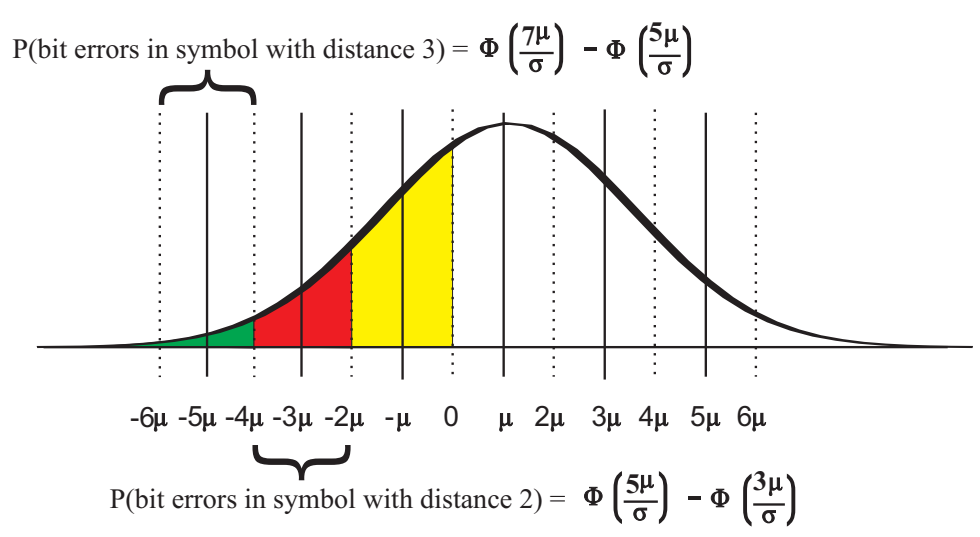

Fig. 7. More Bit Errors

Dependent on the desired accuracy and computational complexity of the BER estimation we can add one or more correction terms to Formula (16). Figure 8 shows the results of these correction factors. The line with the label "2 terms" is the result of the simulation for the original Formula (16) plus the correction term for received neighbors with distance 2 . The line with the label " 3 terms" shows the performance of the BER estimation with Formula (16) plus the correction factors for received neighbors with distance 2 and 3 . Since the Viterbi decoder can not correct frames with a high BER, it is not usefull to add the correction term(s) in our case. 
Figure 8 shows that the error of the BER estimation algorithm is smaller than $2 \%$. Additional simulations for different channels gave a similar result.

Table 3. 64QAM Constellation Bit Encoding

\begin{tabular}{llll|llllll}
000100 & 001100 & 011100 & 010100 & $\mathbf{7}$ & 110100 & 111100 & 101100 & 100100 \\
000101 & 001101 & 011101 & 010101 & $\mathbf{5}$ & 110101 & 111101 & 101101 & 100101 \\
000111 & 001111 & 011111 & 010111 & $\mathbf{3}$ & 110111 & 111111 & 101111 & 100111 \\
000110 & 001110 & 011110 & 010110 & $\mathbf{1}$ & 110110 & 111110 & 101110 & 100110 \\
\hline $\mathbf{- 7}$ & $\mathbf{- 5}$ & $\mathbf{- 3}$ & $\mathbf{- 1}$ & & $\mathbf{1}$ & $\mathbf{3}$ & $\mathbf{5}$ & $\mathbf{7}$ \\
\hline 000010 & 001010 & 011010 & 010010 & $\mathbf{- 1}$ & 110010 & 111010 & 101010 & 100010 \\
000011 & 001011 & 011011 & 010011 & $\mathbf{- 3}$ & 110011 & 111011 & 101011 & 100011 \\
000001 & 001001 & 011001 & 010001 & $\mathbf{- 5}$ & 110001 & 111001 & 101001 & 100001 \\
000000 & 001000 & 011000 & 010000 & $\mathbf{- 7}$ & 110000 & 111000 & 101000 & 100000
\end{tabular}

64QAM BER estimation error with different accuracy for HiperLAN2, AWGN

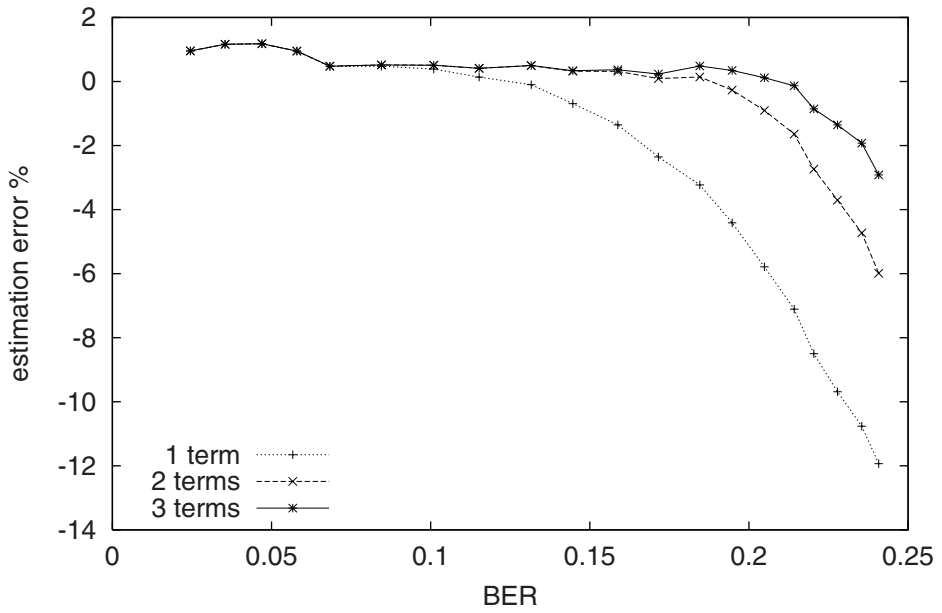

Fig. 8. BER Estimation Error with Different Number of Terms for 64QAM in HiperLAN/2 Simulation with a AWGN Channel

\section{Discussion}

This section dicusses the implementation of the presented in hardware and the advantages of using this method. 


\subsection{Implementation}

The basic idea of the proposed method is to express the quality of the channel (BER) in terms of a normal probability function. This function can be read from a z-table, or certain functions exist that approximate the c.d.f. of a standard normal distribution. For example, $P(x)=1-\frac{1}{2}\left(1+c_{1} x+c_{2} x^{2}+c_{3} x^{3}+c_{4} x^{4}\right)^{-4}+$ $\epsilon(x)$, with $|\epsilon|<2.5 * 10^{-4}[2]$.

The estimators for the $\mu$ and the $\sigma$ of the BER (Formulas 14 and 15 require the summation of $W_{i}^{2}$ and $W_{i}^{4}$ of the incoming bits. This computation has to be done at the incoming bit rate. These computations can be done with very simple hardware support. Because the incoming values of the receiver are always quantized with a limited number of bits, the values of the power of two and four can be stored in a look-up table (LUT). This LUT in combination with an adder and a register are sufficient to compute the sum. The rest of the computation has to be performed only once per frame and can be done e.g. with a general purpose processor.

The estimators do not contain a correction factor for the bias. When the number of samples is large enough $(>30)$, the difference is so small that this can be neglected. Due to the high bit rate (Mbit/s) the bias is not an issue in our case.

\subsection{Advantages of the Presented Method}

The presented method has several attractive properties, such as:

- simplicity - The presented method requires little computation and is therefore easy to implement in a receiver.

- accuracy - Simulations show that the accuracy is within $2 \%$. The method uses all received data symbols instead of only pilot symbols. This means that more data is available which improves the statistical analysis. Furthermore, most methods make an estimation of the BER of the BER of the pilot symbols, which can differ from the BER of the data symbols.

- low overhead - No pilot symbols are used so that all symbols can be used for transmission of data.

- parameter prediction possible - Beside the possiblitiy to predict the BER for the current situation, it is also possible to predict the BER after changes of parameters. For example, it is possible to predict the BER when the modulation is changed.

- no assumptions about environment - Some analytical methods use assumptions about the environment to make a model for prediction of the BER. The presented method does not make assumptions about the environment.

- generality - The presented method is not only useful for HiperLAN/2, but also for other wireless communication methods. A detailed example for wideband code division multiple access (WCDMA) is given in 99. WCDMA differs significantly from the OFDM transmission technology used in Hiper$\mathrm{LAN} / 2$. 


\section{Conclusion}

The presented method to estimate the BER in HiperLAN/2 is simple and effective. With a few formulas, we can describe the complete behaviour of the system in terms of quality. The BER estimation requires no overhead (such as pilot symbols) and has an accuracy of about $2 \%$ with respect to the real BER. A key advantage of our method is that we can also predict what will happen with the BER when we consider to change the modulation type.

Acknowledgements. This research is conducted within the Chameleon project (TES.5004) supported by the PROGram for Research on Embedded Systems \& Software (PROGRESS) of the Dutch organization for Scientific Research NWO, the Dutch Ministry of Economic Affairs and the technology foundation STW. We would like to thank dr. W.C.M. Kallenberg for his support.

\section{References}

1. http://www.3gpp.org

2. M. Abramowitz and I. A. Stegun. Handbook of Mathematical Functions. General Publishing Company, Ltd., seventh edition, 1970. ISBN: 0-486-61272-4.

3. J. Cheng and N. C. Beaulieu. Accurate DS-CDMA bit-error probability calculation in Rayleigh fading. IEEE transactions on wireless communications, 1(1):3-15, 2002.

4. E. J. Dudewicz and S. N. Mishra. Modern Mathematical Statistics. John Wilsey \& Sons, Inc., 1988. ISSN: 0271-6232.

5. J. Khun-Jush, P. Schramm, G. Malmgren, and J. Torsner. HiperLAN2: Broadband wireless communications at $5 \mathrm{GHz}$. IEEE Communications Magazine, pages 130136, June 2002.

6. P. S. Mann. Introductory Statistics. John Wiley \& Sons, second edition, 1995. ISBN: 0-471-31009-3.

7. R. K. Morrow. Accurate CDMA BER calculations with low computational complexity. IEEE Transactions on Communications, pages 1413-1417, Nov. 1998.

8. L. T. Smit. Energy-Efficient Wireless Communication. PhD thesis, University of Twente, Dec. 2003. ISBN: 90-365-1986-1.

9. L. T. Smit, G. J. M. Smit, J. L. Hurink, and A. B. J. Kokkeler. Soft output bit error rate estimation for WCDMA. In Proceedings of Personal Wireless Conference 2003, pages 115-124, Sept. 2003. ISBN: 3-540-20123-8; ISSN: 0302-9743.

10. W. Y. Tan and W. C. Chang. Some comparisions of the method of moments and the maximum likelihood in estimating parameters of a mixture of two normal densities. Journal of the American Statistical Association, 67(33):702-708, Sept. 1972.

11. L. F. W. van Hoesel. Design and implementation of a software defined HiperLAN/2 physical layer model for simulation purposes. Master's thesis, University of Twente, Aug. 2002. 